\title{
Impact de l'altitude sur les caractéristiques des systèmes d'élevage extensifs dans le Haut Atlas central, Maroc
}

\author{
Soufiane El Aayadi ${ }^{1, *}$, Abdelilah Araba $^{1}$ et Magali Jouven ${ }^{2}$ \\ ${ }^{1}$ Département des productions et biotechnologies animales, Institut Agronomique et Vétérinaire Hassan II (IAV Hassan II), BP 6202, \\ 10101 Rabat, Madinate Al IrFAne, Maroc \\ ${ }^{2}$ Institut national d'études supérieures agronomiques de Montpellier (Montpellier SupAgro), UMR Selmet, 2 place Viala, 34060 \\ Montpellier Cedex 2, France
}

\begin{abstract}
Résumé - Dans les régions de montagne, la pérennité des systèmes d'élevage extensifs constitue un enjeu socio-économique et écologique majeur. Notre étude a pour objectif d'analyser la diversité des systèmes d'élevage de petits ruminants dans le Haut Atlas central du Maroc, en fonction de l'altitude. Pour cela, des enquêtes ont été réalisées auprès de 90 éleveurs dans trois communes de la province d'Azilal, situées respectivement en étage inférieur de moyenne montagne, étage supérieur de moyenne montagne et haute montagne. En comparant les élevages sur la base de leur commune d'appartenance, nous montrons que l'altitude a un effet significatif sur leur structure et leur conduite, ainsi que sur certains paramètres sociaux comme l'éducation des éleveurs. En réalisant une typologie sur base statistique, nous identifions quatre types d'élevages : deux types plus extensifs et pastoraux aux étages supérieurs et deux autres intensifiés et associés à l'agriculture en étage inférieur, avec une diversité de situations en termes de structures et de pratiques d'élevage. Cette typologie est cohérente avec les contraintes et opportunités propres à chaque étage altitudinal.
\end{abstract}

Mots clés : pastoralisme / montagne / ovin / pratiques / typologie

Abstract - Impact of altitude on the characteristics of extensive livestock farming systems in the Central High Atlas of Morocco. In mountain regions, the sustainability of extensive livestock farming is a major socio-economic and ecological issue. The aim of our study is to analyze the diversity of small ruminant breeding systems in the Central High Atlas mountains of Morocco, as a function of altitude. Interviews were carried out with 90 breeders in three municipalities of the province of Azilal, at three levels: lower mid-mountain, upper mid-mountain and high mountain. A comparison of the farms on the basis of their location (municipality) shows that altitude has a significant effect on their structure and management, as well as on certain social parameters such as the education of the breeders. On the basis of a statistical classification, we identified four types of farms: two more extensive and pastoral types on the upper altitude and two others intensified and associated with agriculture on the lower altitude, with a diversity of situations in terms of structures and livestock farming practices. This typology is consistent with the constraints and opportunities which characterize each altitudinal level.

Keywords: pastoralism / mountain / sheep / practices / typology

\section{Introduction}

Dans les régions arides et semi-arides, les systèmes d'élevage extensifs constituent la principale occupation et source de revenu de la population rurale. L'élevage des petits ruminants est pratiqué avec des animaux rustiques capables de

\footnotetext{
$\overline{\text { *Auteur de correspondance }}$ : elaayadi.s@gmail.com
}

valoriser des ressources fourragères, rares et variables, disponibles sur les terres de parcours, moyennant des déplacements des troupeaux et des éleveurs (Casasús et al., 2012).

$\mathrm{Au}$ Maroc, l'élevage des petits ruminants est étroitement lié aux espaces pastoraux et sylvo-pastoraux qui sont situés dans les zones agroécologiques peu favorables du pays et couvrent une superficie d'environ 53 millions d'hectares. Les systèmes d'élevage sont caractérisés par la diversité génétique des petits ruminants, la diversité des écosystèmes et 
l'importance des effectifs animaux (20,3 millions d'ovins et 5,6 millions de caprins; MAPMDREF, 2017). Les élevages extensifs marocains contribuent à la création d'emplois $(65 \%$ des emplois du secteur de l'élevage) et à l'amélioration des moyens de subsistance des familles rurales puisqu'ils intéressent près de 1,1 million de foyers ruraux (MAPM, 2014). Les zones de montagne marocaines sont caractérisées par l'élevage extensif. Avec une superficie d'environ $150000 \mathrm{~km}^{2}$, soit $21 \%$ du territoire du Maroc, cet écosystème abrite $30 \%$ des exploitations agricoles et l'essentiel du cheptel de petits ruminants $(50 \%$ des ovins et $90 \%$ des caprins) à l'échelle du pays (MAPM, 2014). Ces zones, considérées comme défavorisées à cause des contraintes liées à la topographie, au climat et à l'altitude, présentent une biodiversité qui a pu être valorisée grâce aux savoir-faire des populations locales (Martin et al., 2014).

Plusieurs travaux de recherche ont analysé la diversité des systèmes d'élevage extensifs en fonction des écosystèmes, des stratégies de subsistance des éleveurs, des ressources mobilisées et des évolutions du contexte (Moyen Atlas oriental du Maroc: Boughalmi et al., 2015; Moyen Atlas central: Sraïri et al., 2003; Haut Atlas central: Benjelloun, 2013; Ibnelbachyr et Mounsif, 2011; Alpes françaises: Nettier et al., 2010). Dans ces travaux, les conduites d'élevage documentées sont susceptibles de varier en fonction de l'exposition et de l'altitude; ces paramètres apparaissent comme un facteur déterminant des systèmes d'élevage, mais sans être étudiés en tant que tels. En effet, la variation des conditions écologiques et climatiques selon l'altitude influence la nature et la disponibilité des ressources mobilisables et par conséquent les modes de conduite (Srour, 2006; Nettier et al., 2015). Les changements auxquels sont confrontés les systèmes extensifs pourraient affecter leur durabilité et mettre en péril leur rôle économique, social et écologique. Dans un tel contexte, il convient de caractériser et comprendre la diversité des systèmes d'élevage extensifs pour pouvoir accompagner leurs adaptations et assurer leur durabilité.

Notre étude a porté sur une zone d'élevage pastoral dans l'Atlas marocain. L'objectif était d'analyser la diversité des systèmes d'élevage extensifs en relation avec le contexte. Nous faisons l'hypothèse que l'altitude est un facteur majeur influençant les systèmes d'élevage, via des conditions écologiques mais aussi socio-économiques spécifiques. Pour cela, deux approches complémentaires ont été menées, à partir d'enquêtes auprès de 90 éleveurs : (1) une recherche $a$ priori de différences statistiques dans la structure ou la conduite des élevages en fonction de leur commune d'appartenance, prise comme un indicateur d'altitude; (2) une classification statistique des élevages selon leurs caractéristiques, suivie d'une analyse des groupes ainsi constitués pour identifier les facteurs susceptibles d'expliquer cette diversité, dont notamment l'altitude.

\section{Matériel et méthodes}

\subsection{Zone d'étude}

Notre étude a été menée dans le Haut Atlas central, dans la province d'Azilal. Celle-ci est caractérisée par un climat de type semi-aride au nord et subhumide au sud avec une pluviométrie moyenne de $400 \mathrm{~mm} / \mathrm{an}$, une température moyenne minimale de 4 à $10^{\circ} \mathrm{C}$ et maximale de 20 à $40^{\circ} \mathrm{C}$ (DPA d'Azilal, 2019). La province d'Azilal abrite 554000 habitants dont la majorité sont ruraux $(82 \%$ de la population totale). Cette zone de montagne comporte de vastes superficies de parcours et de forêts ( $52 \%$ de la surface totale) avec une activité agricole dominée par l'élevage extensif des petits ruminants (584000 ovins et 412000 caprins) (MAPMDREF, 2017). La présente étude a concerné trois communes : Ouaoula, Ait M'hamed et Tabant (Fig. 1). Ces communes se situent dans trois étages altitudinaux distincts qui diffèrent par leur climat (Tab. 1) et en particulier par le niveau des précipitations et la rigueur de l'hiver, qui influencent la diversité des ressources pastorales présentes. Elles diffèrent aussi par leur population humaine et de petits ruminants (Fig. 1) et par leur accessibilité, les villages d'altitude ne pouvant être atteints qu'au prix de plusieurs heures de trajet à pied ou à dos de mulet.

\subsection{Enquêtes auprès des éleveurs}

Des entretiens ont été réalisés auprès d'un échantillon d'éleveurs de la province d'Azilal, entre avril et juillet 2019. Ainsi, 90 éleveurs (30 par commune), disponibles et suffisamment accessibles, ont été échantillonnés suivant leur répartition géographique de façon à couvrir un maximum de villages (douars), sachant que les pratiques sont en général homogènes au sein d'un village. Le choix de l'échantillon n'a pas été axé sur une représentativité statistique, l'objectif étant de couvrir une diversité de cas permettant d'identifier les différents systèmes d'élevage et leurs spécificités en lien avec l'altitude et l'accès aux ressources.

Les entretiens ont été semi-directifs (Kauffman, 1996) et individuels. Ils ont été menés, en berbère et en arabe, chez les éleveurs et ont duré chacun $1 \mathrm{~h} 30$ à $2 \mathrm{~h} 30$. Ces entretiens ont porté sur les thèmes suivants (Tab. 2): (1) identification de l'éleveur; (2) caractéristiques de l'exploitation agricole; (3) caractéristiques du troupeau; (4) conduite du troupeau. Les discussions avec chaque éleveur ont fait l'objet d'une prise de notes par écrit et d'un enregistrement vocal pour pouvoir produire a posteriori une transcription exacte des dires d'éleveurs. Les données brutes obtenues ont servi à construire une base de données quantitatives et qualitatives, ces dernières étant organisées en classes.

\subsection{Analyses statistiques}

Une analyse a priori des différences entre exploitations sur la base de leur commune d'appartenance a permis de rechercher les caractéristiques des élevages significativement affectées par l'altitude. Pour ce faire, une analyse de variance (ANOVA) a été appliquée, pour les variables quantitatives, selon le modèle: $\mathrm{Yij}=\mathrm{m}+\mathrm{Cri}+\mathrm{Eij}$, avec :

- Yij: variable analysée;

- m: moyenne globale;

- Cri : effet de la commune $\approx$ altitude $(i=1,2,3)$;

- Eij : terme d'erreur.

Une comparaison multiple de moyennes (deux à deux) par la méthode de Tukey a ensuite permis de rechercher les 


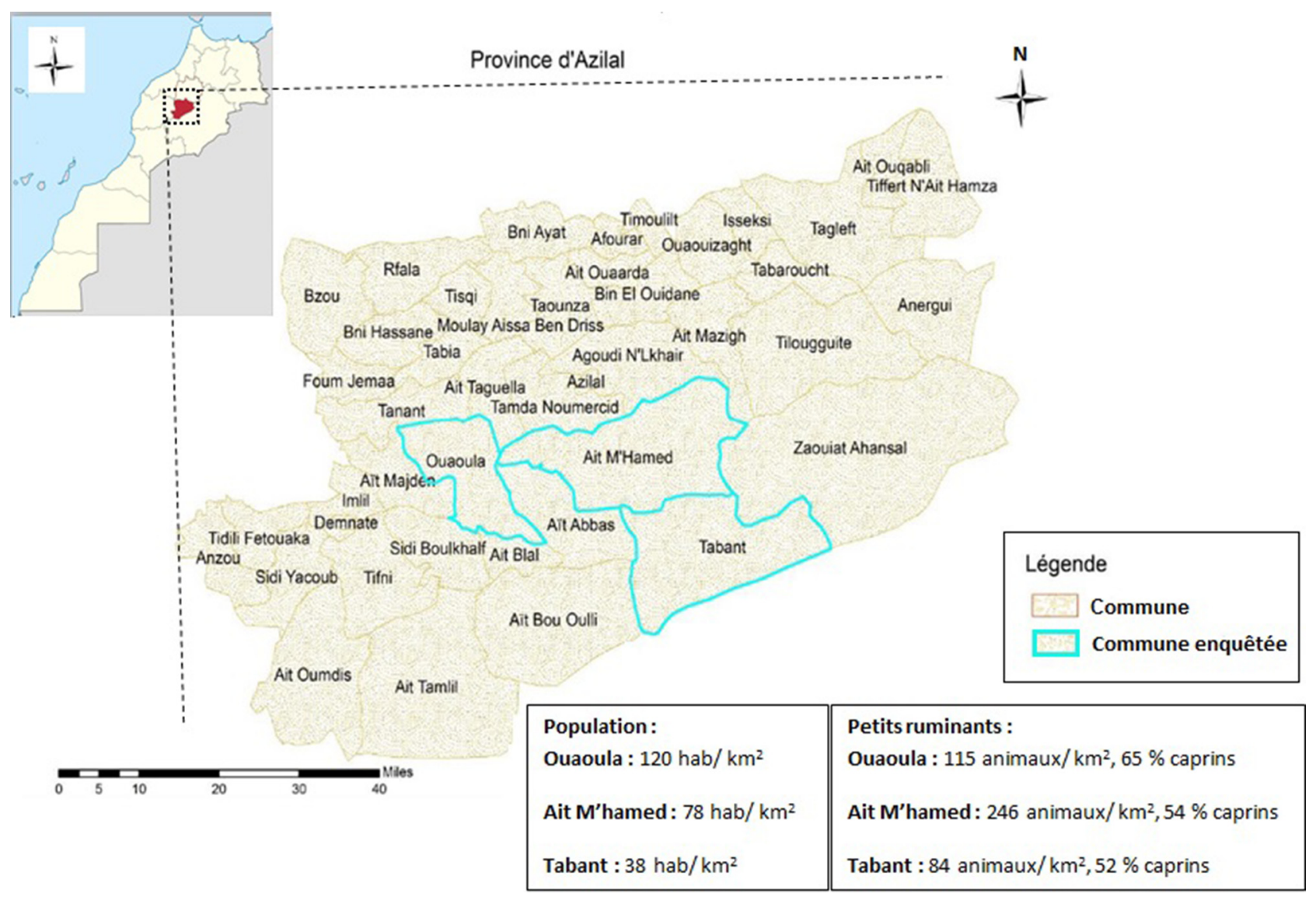

Fig. 1. Localisation de la zone d'étude (source: CRI de Béni Mellal-Khénifra, 2015; DPA d'Azilal, 2019).

Fig. 1. Localization of the study area.

Tableau 1. Caractéristiques climatiques des sites retenus pour l'étude.

Table 1. Climatic characteristics of the sites selected for the study.

\begin{tabular}{llll}
\hline Étage & Moyenne montagne, étage inférieur & Moyenne montagne, étage supérieur & Haute montagne \\
\hline Commune & Ouaoula & Ait M'hamed & Tabant \\
Altitude & $800 \mathrm{~m}-1400 \mathrm{~m}$ & $1400 \mathrm{~m}-1800 \mathrm{~m}$ & $1800 \mathrm{~m}-3000 \mathrm{~m}$ \\
Bioclimat & Semi-aride & Semi-aride à subhumide & Semi-aride à subhumide \\
Précipitations & $400 \mathrm{~mm} / \mathrm{an}$ & $500 \mathrm{~mm} / \mathrm{an}$ & $600 \mathrm{~mm} / \mathrm{an}$ \\
moyennes & & & Hiver très froid avec des \\
Climat & Hiver tempéré à froid & Hiver très froid avec & chutes de neige \\
& avec des sécheresses fréquentes & des chutes de neige & \\
\hline
\end{tabular}

différences significatives $(p<0,05)$ entre communes. Pour les variables qualitatives, des tests du $\chi^{2}$ ont été appliqués pour comparer les communes $(p<0,05)$.

Une analyse a posteriori de l'ensemble des élevages étudiés, sans tenir compte de la commune, a été réalisée avec des statistiques multivariées pour identifier des groupes d'élevages. Dans un premier temps, une analyse factorielle multiple (AFM) a permis de réduire les 26 variables initiales à 13 variables et 22 modalités synthétisant plus de $85 \%$ de l'information (Tab. 2). Celles-ci ont servi pour une Classification hiérarchique en composantes principales (HCPC) en vue d'identifier des groupes d'élevage présentant des caractéristiques communes. Enfin, une ANOVA a été effectuée selon le modèle: $Y i j=m+$ Tri + Eij, avec :

- Yij: variable analysée;

- m: moyenne globale;

- Tri : effet du groupe d'élevage $(i=1,2,3,4)$;

- Eij: terme d'erreur.

Elle a été suivie d'une comparaison multiple de moyennes par la méthode de Tukey (pour les variables quantitatives) 
Tableau 2. Variables étudiées et variables retenues pour l'analyse factorielle multiple.

Table 2. Variables studied and used for the multiple factor analysis.

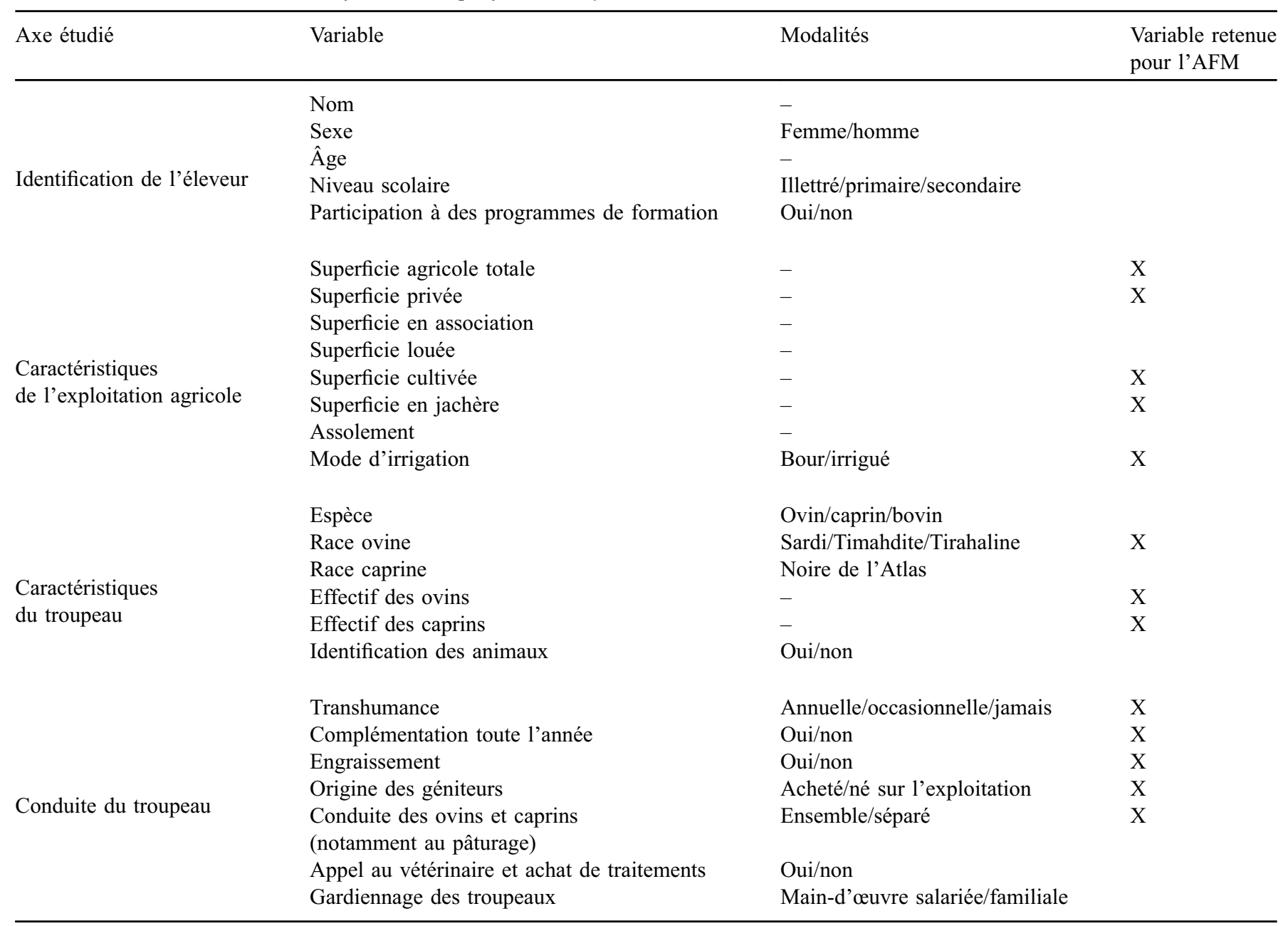

et des tests du $\chi^{2}$ (pour les variables qualitatives), ont été appliqués pour rechercher les différences significatives $(p<0,05)$ entre groupes d'élevage.

Toutes les analyses statistiques ont été réalisées avec le logiciel $\mathrm{R}^{\odot}$ ( $\mathrm{R}$ core team, 2019).

\section{Résultats}

\subsection{Caractéristiques de l'échantillon étudié et différences entre communes}

Parmi les éleveurs interrogés, la grande majorité (92\%) sont des hommes, et l'âge moyen est de 50 ans. Les deux-tiers $(67 \%)$ des éleveurs sont illettrés, $13 \%$ ont une éducation de niveau primaire et $20 \%$ de niveau secondaire. L'instruction des éleveurs diffère entre communes $(p<0,05)$, avec un taux d'illettrisme plus élevé à Tabant ( $80 \%$ des éleveurs interrogés) et un taux d'instruction plus élevé à Ouaoula $(48 \%)$ et Ait M'hamed (32\%). Le niveau d'insertion des éleveurs dans des associations locales (47\%) est limité, malgré les incitations nationales. La majorité $(90 \%)$ des éleveurs à Ouaoula participe activement aux formations assurées par les services agricoles locaux, contre 60 et $30 \%$ des éleveurs respectivement à Ait M'hamed et Tabant $(p<0,05)$.
La conduite des animaux est réalisée par la main-d'œuvre familiale, exclusive dans $87 \%$ des cas. La main-d'œuvre salariée concerne les grands éleveurs ( $>100$ ovins et caprins) et parfois, lors des périodes de transhumance, ceux disposant d'effectifs réduits qui s'associent pour atteindre 300 à 400 têtes de petits ruminants et embauchent un berger.

La superficie agricole totale (SAT) exploitée est en moyenne de 11,4 ha par exploitation, avec une grande disparité entre éleveurs. La SAT moyenne (Tab. 3) est significativement moins importante à Tabant $(p<0,05)$, où la majorité des terres sont privées (contre seulement $2 / 3$ ailleurs; $p<0,05$ ) et fortement morcelées (le nombre de parcelles exploitées varie de 5 à 14). La SAT moyenne varie entre 2,5 et 60 ha à Ouaoula et entre 4 et 40 ha à Ait M'hamed, avec une grande hétérogénéité de répartition du patrimoine foncier. La part de la superficie cultivée est beaucoup plus importante à Tabant $(98 \%)(p<0,05)$. Elle y est en majorité $(80 \%)$ irriguée, alors que l'irrigation ne concerne que $53 \%$ de la SAT à Ait M'hamed. La totalité de la superficie cultivée à Ouaoula dépend du régime pluvial $(p<0,05)$. La SAT non cultivée correspond à des jachères pâturées. Contrairement aux autres communes où les terres cultivées sont dispersées, à Tabant les cultures se concentrent dans les fonds de la vallée Ait Bougemaz, où elles sont irriguées par dérivation des eaux de rivières. 
Tableau 3. Caractéristiques des exploitations agricoles dans les communes étudiées.

Table 3. Characteristics of the farms in the municipalities studied.

\begin{tabular}{lccc}
\hline Caractéristiques des communes & Ouaoula & Ait M'hamed & Tabant \\
\hline Superficie agricole totale (ha) & $19,9 \pm 14,9 \mathrm{a}$ & $10,4 \pm 10,0 \mathrm{~b}$ & $4,0 \pm 1,2 \mathrm{c}$ \\
Superficie privée (ha) & $13,8 \pm 15,2 \mathrm{a}$ & $6,4 \pm 7,5 \mathrm{~b}$ & $4,0 \pm 1,2 \mathrm{c}$ \\
Superficie en association (ha) & $3,2 \pm 6,0 \mathrm{a}$ & $3,1 \pm 7,0 \mathrm{a}$ & $0,0 \pm 0,0 \mathrm{~b}$ \\
Superficie louée (ha) & $2,9 \pm 5,3 \mathrm{a}$ & $0,9 \pm 1,5 \mathrm{~b}$ & $0,0 \pm 0,0 \mathrm{~b}$ \\
Superficie cultivée (ha) & $11,4 \pm 8,8 \mathrm{a}$ & $4,6 \pm 3,4 \mathrm{~b}$ & $3,9 \pm 1,2 \mathrm{~b}$ \\
Superficie en jachère (ha) & $8,5 \pm 7,8 \mathrm{a}$ & $5,8 \pm 7,7 \mathrm{a}$ & $0,1 \pm 0,1 \mathrm{~b}$ \\
\hline
\end{tabular}

Les moyennes affectées d'une lettre différente diffèrent significativement $(P<0,05)$.

Tableau 4. Effectifs moyens des troupeaux dans les communes étudiées.

Table 4. Average size of herds in the municipalities studied.

\begin{tabular}{lccc}
\hline Espèce & Ouaoula & Ait M'hamed & Tabant \\
\hline Ovine (têtes) & $54 \pm 24 \mathrm{a}$ & $83 \pm 66 \mathrm{~b}$ & $92 \pm 53 \mathrm{c}$ \\
Caprine (têtes) & $23 \pm 34 \mathrm{a}$ & $32 \pm 30 \mathrm{a}$ & $44 \pm 50 \mathrm{a}$ \\
Bovine (têtes) & $2 \pm 1 \mathrm{a}$ & $2 \pm 1 \mathrm{a}$ & $1 \pm 1 \mathrm{a}$ \\
\hline
\end{tabular}

Les moyennes affectées d'une lettre différente diffèrent significativement $(P<0,05)$.

Les éleveurs interrogés pratiquent aussi l'agriculture, avec (du plus au moins fréquent, en nombre d'hectares): céréales, arboriculture, fourrages, cultures maraîchères et légumineuses. Les types de cultures dominants sont les mêmes dans toute la zone d'étude. Les cultures céréalières (orge, blé dur et blé tendre) occupent en moyenne $82 \%$ de la SAT, car elles nécessitent peu d'investissements et s'accommodent du climat local. L'arboriculture occupe en moyenne $10 \%$ de la SAT; elle est dominée par l'olivier et l'amandier (souvent associé aux céréales) à Ouaoula et par le pommier et le noyer à Ait M'hamed et Tabant. Les cultures fourragères, représentées principalement par la luzerne (souvent cultivée sous pommiers), caractérisent les communes de Tabant et Ait M'hamed. Elles occupent un peu moins de $5 \%$ de la SAT. Les cultures maraîchères (pomme de terre et oignons) à Tabant et Ait M'hamed, et les légumineuses (lentille, pois, fève), dans les trois communes, occupent 2 et $1 \%$ de la SAT, respectivement. Elles sont en grande partie destinées à l'alimentation humaine et dans de rares cas aux animaux (fanes des cultures maraîchères en période de soudure et fèves en période d'engraissement pour l'Aïd El Adha).

Le nombre d'unités gros bétail (UGB) totales exploitées par les 90 éleveurs interrogés est de 1600 (en comptant 1 UGB pour 7 ovins ou caprins; CIRAD-GRET, 2009). Les ovins représentent $64 \%$ des UGB totales, avec une moyenne de 11,4 UGB par éleveur, suivis des caprins et des bovins avec des moyennes respectives de 4,9 et 1,4 UGB par éleveur (28 et $8 \%$ des UGB totales, respectivement). L'élevage des ovins est pratiqué par la totalité des éleveurs, alors que l'élevage des caprins et des bovins est présent dans 59 et $70 \%$ des élevages, respectivement. L'espèce bovine est conduite en petits troupeaux laitiers de type croisé dont le lait est destiné principalement à l'autoconsommation. L'effectif moyen d'ovins par éleveur est plus élevé à Tabant $(P<0,05)$, mais les effectifs caprins et bovins ne diffèrent pas significativement entre communes (Tab. 4). Les troupeaux ovins sont essentiellement composés d'animaux de phénotype Sardi à Ouaoula (100\% des éleveurs), de phénotype Timahdite à Ait M'hamed (100\% des éleveurs) et à Tabant ( $87 \%$ des éleveurs) et de la population de montagne Tirahaline chez $13 \%$ des éleveurs à Tabant. Les effectifs de caprins sont très variables entre exploitations (33 en moyenne, variant de 0 à 140) et dominés par la population Noire de l'Atlas.

La mobilité des éleveurs et des troupeaux dans la province d'Azilal varie d'une commune à l'autre. Elle est conditionnée par la disponibilité des ressources alimentaires sur parcours, l'importance des effectifs d'animaux et les races élevées. La majorité des éleveurs sont des transhumants, selon deux modalités :

- la transhumance optionnelle, très répandue à Ouaoula, est pratiquée par $70 \%$ des éleveurs interrogés dans cette commune (contre 33 et $37 \%$ des éleveurs respectivement à Ait M'hamed et Tabant); elle consiste à pratiquer une mobilité à courte distance $(<50 \mathrm{~km})$ du troupeau si les ressources fourragères des parcours de proximité font défaut;

- la transhumance annuelle, pratiquée par 37 et $50 \%$ des éleveurs respectivement à Ait M'hamed et Tabant; elle consiste à intégrer une mobilité plus longue et d'amplitude plus importante $(>100 \mathrm{~km})$ du troupeau chaque année, quelles que soient les ressources disponibles sur les parcours de proximité $(p<0,05)$.

Il existe également des éleveurs sédentaires exploitant des parcours privés (à Ouaoula et Ait M'hamed) ou collectifs (toutes les communes), près des lieux d'habitation.

Les ovins et caprins sont conduits ensemble au pâturage toute l'année chez $80 \%$ des éleveurs à Ait M'hamed et chez la totalité des éleveurs à Tabant; ils sont conduits séparément 
Tableau 5. Caractéristiques des quatre types d'élevage identifiés par classification hiérarchique.

Table 5. Characteristics of the four types of breeding systems identified with a hierarchical classification.

\begin{tabular}{|c|c|c|c|c|}
\hline Type d'élevage & $\begin{array}{l}\text { Petits éleveurs } \\
\text { sédentaires avec } \\
\text { de moyennes propriétés }\end{array}$ & $\begin{array}{l}\text { Eleveurs moyens semi- } \\
\text { sédentaires avec } \\
\text { de grandes propriétés }\end{array}$ & $\begin{array}{l}\text { Petits éleveurs à } \\
\text { faible mobilité avec de } \\
\text { petites propriétés }\end{array}$ & $\begin{array}{l}\text { Grands éleveurs à } \\
\text { forte mobilité avec de } \\
\text { moyennes propriétés }\end{array}$ \\
\hline Nombre d'élevages & 9 & 21 & 33 (18 et 15$)$ & $27(12$ et 15$)$ \\
\hline Superficie totale (ha) & $10,5 \pm 3,1 \mathrm{a}$ & $23,9 \pm 6,4 b$ & $3,7 \pm 1,1 \mathrm{c}$ & $11,6 \pm 5,0 \mathrm{~d}$ \\
\hline Superficie cultivée (ha) & $6,0 \pm 2,8 \mathrm{a}$ & $13,3 \pm 6,2 b$ & $2,7 \pm 1,1 \mathrm{c}$ & $6,2 \pm 1,2 \mathrm{a}$ \\
\hline Effectif des ovins (têtes) & $31 \pm 7 \mathrm{a}$ & $64 \pm 17 b$ & $49 \pm 22 c$ & $135 \pm 38 d$ \\
\hline Effectif des caprins (têtes) & $3 \pm 1 \mathrm{a}$ & $31 \pm 15 b$ & $16 \pm 6 c$ & $65 \pm 25 d$ \\
\hline Race des ovins & Sardi & Sardi & Timahdite & Timahdite et Tirahaline \\
\hline \multirow{2}{*}{$\begin{array}{l}\text { Type de mobilité } \\
\text { (\% éleveurs) }\end{array}$} & 100 & 0 & 38 & 0 \\
\hline & 0 & 100 & 62 & 0 \\
\hline $\begin{array}{l}\text { Complémentation toute } \\
\text { l'année }(\% \text { éleveurs) } *\end{array}$ & 100 & 100 & 64 & 18 \\
\hline
\end{tabular}

Les moyennes affectées d'une lettre différente diffèrent significativement $(P<0,05)$.

* Des différences significatives entre les groupes identifiés ont été détectées au seuil de $5 \%$ par le test de $\chi^{2}$.

** La commune n'a pas été prise en compte pour la classification statistique des élevages.

chez $94 \%$ des éleveurs à Ouaoula $(p<0,05)$. Pour les petits ruminants, la totalité des éleveurs pratiquent une lutte libre donnant lieu à des naissances étalées. Les femelles de renouvellement sont toujours issues du troupeau. Pour les géniteurs mâles, si $90 \%$ des éleveurs de Ouaoula se fournissent au marché, cela est rare dans les autres communes $(p<0,05)$, où $80 \%$ ou plus des éleveurs gardent des mâles nés sur l'exploitation. La complémentation systématique, toute l'année, des animaux, et en particulier des ovins, est pratiquée par la totalité des éleveurs de Ouaoula, contre 60 et $27 \%$ des éleveurs respectivement à Ait M'hamed et Tabant $(p<0,05)$. Pour les autres, la complémentation est stratégique, réservée aux périodes de forts besoins ou de disponibilité fourragère insuffisante sur les parcours (périodes de lutte et d'agnelage, et lors de fortes chutes de neige entre décembre et février). De même, l'engraissement des animaux est pratiqué par tous les éleveurs de Ouaoula, contre 60 et $40 \%$ respectivement à Ait M'hamed et Tabant $(p<0,05)$. Les pratiques de complémentation et d'engraissement sont basées sur l'utilisation des ressources produites sur l'exploitation (chaumes et paille de céréales et de légumineuses, orge grain pour tous les étages et luzerne verte ou foin et résidus de cultures maraîchères pour les étages supérieurs), ou bien achetées (orge grain et son de blé) et/ou issues des forêts (feuillages, branches d'arbre, glands). Enfin, les éleveurs qui font appel au vétérinaire pour traiter leurs animaux sont rares et ne représentent que 17, 7 et $3 \%$ de l'ensemble respectivement à Ouaoula, Ait M'hamed et Tabant $(p<0,05)$. Les traitements traditionnels et l'achat de médicaments sans ordonnance dominent, en complément des campagnes de prophylaxie réalisées par l'État.

\subsection{Classification statistique des élevages étudiés}

L'AFM et l'HCPC ont permis de distinguer quatre types d'élevage. L'axe 1 du plan factoriel de l'AFM explique 40,5\% de la variabilité totale; il oppose les élevages transhumants disposant d'un cheptel de petits ruminants important, à des élevages sédentaires et semi-sédentaires disposant d'effectifs ovins et caprins moins importants. L'axe 2 explique $35,6 \%$ de la variabilité totale et oppose des éleveurs possédant beaucoup de surfaces en propriété à d'autres disposant de petites propriétés. L'axe 3 explique seulement $10 \%$ de la variabilité totale et rend compte de la présence de l'activité d'engraissement chez les éleveurs possédant des propriétés moyennes à grandes. L'HCPC nous a permis de distinguer 4 grands types d'élevage, décrits ci-après (Tab. 5).

Deux types se retrouvent à l'étage inférieur (Ouaoula), reposant fortement sur la complémentarité avec l'agriculture et exploitant la race ovine Sardi complémentée toute l'année à partir des aliments autoproduits et/ou achetés. Ces types diffèrent par la taille des surfaces exploitées (hors parcours) et des troupeaux $(p<0,05)$. Le premier type d'élevage, «petits éleveurs sédentaires avec de moyennes propriétés", est composé de 9 éleveurs qui détiennent de petits effectifs de petits ruminants (en moyenne 31 ovins et 3 caprins), le plus souvent conduits séparément (chez les $2 / 3$ des éleveurs). La SAT exploitée est en moyenne de 10,5 ha, dont 6 ha (privés) sont cultivés en pluvial et le reste (terres louées ou en association) est réservé au pâturage des animaux. Le deuxième type, «éleveurs moyens semi-sédentaires avec de grandes propriétés », est composé de 21 éleveurs possédant un effectif 
moyen de 64 ovins et 31 caprins, conduits séparément chez la totalité des éleveurs. Ces derniers pratiquent, lors des périodes de sécheresse ou lorsque les ressources fourragères des parcours de proximité deviennent très rares (début février à mimai), une transhumance optionnelle à courte distance vers le piémont au nord $(<50 \mathrm{~km})$. De plus, la SAT est nettement supérieure à celle exploitée par les autres types (23,9 ha en moyenne, dont 13,3 ha cultivés en pluvial; $p<0,05)$. Les élevages de l'étage inférieur (types 1 et 2) pratiquent tous l'engraissement des mâles lors des périodes de l'Aïd El Adha.

Deux autres types caractérisent les étages supérieurs (Ait M'hamed et Tabant). L'un est composé de 33 «petits éleveurs à faible mobilité avec de petites propriétés», qui pratiquent l'élevage en complément d'autres activités (commerce et tourisme). Ces éleveurs possèdent en moyenne 49 ovins de race Timahdite et 16 caprins, souvent conduits ensemble, et exploitent une SAT très réduite ( 3,7 ha en moyenne), en majorité irriguée et cultivée $(p<0,05)$. Les ressources fourragères de l'exploitation (luzerne verte ou foin, paille de céréales et de légumineuses, chaumes, résidus de cultures maraîchères) et des parcours collectifs de proximité (forêt comprise) constituent l'essentiel de l'alimentation des animaux. La majorité des éleveurs complémentent les animaux toute l'année. En plus, les plus gros éleveurs pratiquent une transhumance optionnelle à courte distance $(<50 \mathrm{~km})$ vers les parcours d'altitude (en été) lors des périodes de soudure. La totalité de ces éleveurs sont des naisseurs et vendent leurs animaux après le sevrage, au fur et à mesure et selon les besoins de la famille. L'autre type, «grands éleveurs à forte mobilité avec de moyennes propriétés», est composé de 27 élevages disposant d'importants effectifs de petits ruminants : en moyenne 135 ovins de races Timahdite et Tirahaline et 65 caprins $(p<0,05)$, souvent conduits ensemble. La SAT est en moyenne de 11,6 ha, dont 6,2 ha irrigués et cultivés, le reste étant réservé au pâturage. Cette superficie agricole est loin de satisfaire aux besoins des cheptels. Par conséquent, les éleveurs de ce groupe, en plus des parcours de proximité, pratiquent annuellement des transhumances longues $(>100 \mathrm{~km})$ pour accéder à des parcours d'hiver (vers le sud) et à des parcours d'altitude (haute montagne) en été. Les aliments produits sur l'exploitation et achetés sont utilisés uniquement pour complémenter les animaux lors des périodes de soudure ou d'agnelage et pour l'engraissement des mâles lors des périodes de l'Aïd Al Adha.

\section{Discussion}

Notre étude a porté sur une province située dans une zone montagneuse, hostile et difficilement aménageable en comparaison avec les zones de plaine. L'impact de l'altitude sur les activités d'élevage est un élément original, qui n'avait pas été abordé dans les précédents travaux de recherche sur l'élevage en montagne au Maroc (Sraïri et al., 2003; Ibnelbachyr et Mounsif, 2011). En comparant les élevages sur la base de leur commune d'appartenance (et donc de l'altitude de celle-ci), nous avons montré que l'altitude a un effet significatif sur leur structure et leur conduite, ainsi que sur certains paramètres sociaux comme l'éducation des éleveurs. En réalisant une typologie sur base statistique, nous avons identifié des systèmes d'élevage et observé deux dichotomies: d'une part, entre systèmes d'élevage intensifiés et associés à l'agriculture en étage inférieur et systèmes plus extensifs et pastoraux aux étages supérieurs et d'autre part, pour un étage donné, entre petits et grands cheptels.

Quelle que soit la méthode d'analyse, on constate un impact significatif de l'étage altitudinal sur les caractéristiques des élevages. En étage inférieur de moyenne montagne (Ouaoula), des cheptels de petite taille sont associés à d'importantes surfaces de cultures céréalières en régime pluvial, avec deux systèmes d'élevage majoritaires et une diversité de situations en termes de foncier et de pratiques. En haute montagne (Tabant), les exploitations sont globalement semblables, avec d'importants cheptels très mobiles conduits sur parcours et des surfaces cultivées limitées, en irrigué. La situation est intermédiaire en étage supérieur de moyenne montagne (Ait M'hamed). En haute montagne, les pentes sont importantes, les sols pauvres et les fréquents orages et crues causent des inondations. Dans ces conditions contraignantes, les éleveurs n'ont pas beaucoup d'options en termes de structure et de conduite de leur ferme. Des situations similaires ont été rapportées dans les Andes centrales (FAO, 2010) et dans les Pyrénées centrales (Gibon et al., 2004).

L'enclavement, accentué par l'altitude, constitue une contrainte majeure ralentissant le développement du secteur de l'élevage dans la province d'Azilal (El Amiri et al., 2007). Ainsi, la majorité des villages des éleveurs interrogés à Tabant, et dans une moindre mesure à Ait M'hamed, sont à une distance de 1 à 5 heures à pied ou à dos de mulet du centre des communes. De ce fait, l'accès aux services (agricoles, commerciaux, écoles, vétérinaires...) est limité. Cela peut affecter les conduites d'élevage, car le taux élevé d'illettrisme entraîne un faible niveau de participation aux associations locales. Le rôle de celles-ci se limite généralement à organiser les campagnes de vaccination et de réception de subventions étatiques, mais donne aussi parfois accès à des sessions de formation assurées par les services agricoles. Lors de la phase d'enquête, nous avons pu observer que les éleveurs de Ouaoula étaient plus aptes à assister aux formations assurées par les services agricoles locaux, à comprendre leurs contenus et à appliquer les recommandations, en comparaison avec les éleveurs des autres communes étudiées. L'enclavement exclut la grande majorité des éleveurs de la zone d'étude d'un encadrement par des organisations professionnelles (comme l'ANOC, Association nationale ovine et caprine). L'isolement rend difficile l'approvisionnement en aliments du bétail, l'achat de géniteurs et la commercialisation des animaux engraissés. L'impact négatif de l'enclavement sur l'instruction et l'encadrement technique a été rapporté dans le Moyen Atlas oriental par Nassif et al. (2011). Dans les Alpes françaises, Amoudry (2002) a observé en haute montagne que l'isolement, l'inaccessibilité des exploitations et la dispersion spatiale des troupeaux limitent la diffusion du progrès technique par les services de développement agricole et engendre d'importants surcoûts dans l'achat d'intrants, en particulier d'aliments. Gibon et al. (2004) ont souligné aussi que les caractéristiques spatiales de l'exploitation influent sur la maîtrise des conduites dans les Pyrénées centrales. Ces auteurs ont étudié des zones de montagne où, comme dans la province d'Azilal, une grande partie des terrains des exploitations se situent en altitude avec une dispersion des unités de gestion, un fort étagement et des difficultés d'accès. 
Comme pour la majorité des zones montagneuses au Maroc et dans le monde, les parcours sont essentiels à l'alimentation des petits ruminants et leur valorisation est permise par la mobilité du troupeau et de l'éleveur. Ce mode d'élevage est très répandu dans les zones de haute montagne du Maroc, avec toutefois une régression depuis deux décennies (Alary et al., 2019) en raison de la dégradation des parcours, de la rareté de la main-d'œuvre et du désintérêt des jeunes générations (Boughalmi et al., 2015). A l'image de ce qui a été rapporté par Srour (2006) au Liban et par Gavinet (2007) dans le Haut Atlas, la transhumance annuelle reste très pratiquée lorsque les cheptels sont importants, quel que soit l'état des parcours de proximité. Cette mobilité est favorisée par la présence de races adaptées aux parcours pentus et accidentés et par l'association d'ovins et de caprins qui permet l'exploitation d'une large gamme de ressources pastorales (Srour, 2006). Les races ovines élevées à Tabant et à Ait M'hamed (Timahdite et Tirahaline) sont capables d'exploiter les mêmes types de parcours que les caprins (parcours accidentés et forêts avec des pentes accentuées), contrairement à Ouaoula où les ovins Sardi ne sont pas adaptés aux terrains pentus et accidentés, valorisés par les caprins seuls. Les cheptels importants et la difficulté de trouver de la main-d'œuvre obligent les éleveurs à garder les deux espèces ensemble pour limiter la charge de travail. A des altitudes plus faibles ou avec des cheptels modérés, la transhumance est pratiquée uniquement lorsque les ressources des parcours de proximité sont limitées. Des formes d'élevage sédentaire dominent dans le cas de petits cheptels, comme l'ont déjà rapporté Bourbouze (1999) et Srour (2006), quelles que soient l'altitude et les races élevées.

Les étages altitudinaux étudiés présentent un contexte différent, conduisant à une diversité de systèmes d'élevage. La différence est particulièrement prononcée entre l'étage inférieur (Ouaoula) et les étages supérieurs (Ait M'hamed et Tabant). Comme indiqué plus haut, les contraintes physiques (l'enclavement) et leurs conséquences en termes de contraintes sociales et d'accompagnement technique s'accentuent avec l'altitude, et par conséquent impactent inégalement la structure des exploitations et la conduite d'élevage au niveau de chaque étage altitudinal. Par ailleurs, la contrainte hydrique marque l'étage inférieur, limitant ainsi les possibilités de diversification culturale (fourrages irrigués, arbres fruitiers, cultures maraîchères) comparé aux altitudes élevées. Il convient de souligner qu'en général, les systèmes d'élevage extensif dans la province d'Azilal doivent composer, en plus des contraintes précitées, avec des contraintes climatiques fortes impactant la disponibilité des ressources pastorales dans les différents étages altitudinaux. En effet, la réduction de la dimension pastorale a marqué les systèmes d'élevage de basse altitude possédant des superficies agricoles et des moyens financiers importants. Dans ces systèmes, le recours aux aliments achetés ou autoproduits est devenu essentiel, comme ailleurs au Maghreb (Jemaa et al., 2016; Bourbouze, 2006), pour complémenter toute l'année des effectifs faibles à modérés de petits ruminants. En étages supérieurs, l'activité de l'élevage dépend fortement des ressources pastorales, encore disponibles, notamment chez les grands éleveurs possédant de moyennes propriétés. Cette activité (aux dires des éleveurs interrogés) est précaire et ne peut pas couvrir les besoins familiaux des petits éleveurs avec de petites propriétés. Ces derniers exercent d'autres activités extra-agricoles pour maintenir la viabilité de l'exploitation.

La dynamique des systèmes d'élevage identifiés et leurs modalités d'adaptation face à la disponibilité des ressources pastorales mériteraient d'être plus minutieusement étudiées pour pouvoir identifier les facteurs de résilience et proposer des moyens pour accompagner le changement dans ces systèmes et in fine assurer leur durabilité.

\section{Conclusion}

Cette étude constitue une étape préliminaire qui vise à caractériser la diversité des systèmes d'élevage de petits ruminants en fonction de l'étagement altitudinal. Elle a permis d'identifier l'effet de l'altitude aussi bien sur les paramètres sociodémographiques de la population locale, peu étudiés dans la majorité des travaux réalisés au Maroc, que sur la structure des élevages et sur les conduites d'élevage des petits ruminants.

Notre étude a montré que les contraintes physiques, sociales et d'accompagnement technique s'accentuent avec l'altitude. En haute montagne, on retrouve des systèmes d'élevage caractérisés par de plus faibles surfaces cultivées, des troupeaux de petits ruminants plus grands, une mobilité systématique et un moindre recours à la complémentation, par rapport aux systèmes d'élevage à plus faible altitude. L'enclavement et la rigueur climatique sont à l'origine de ces différences. Ces contraintes apparaissent en effet réparties selon un gradient altitudinal, avec cependant plusieurs conduites possibles au sein d'un même étage. Ces résultats, mis en évidence pour la province d'Azilal dans le Haut Atlas central, pourraient s'appliquer aux autres zones de montagne du Maroc au vu de la ressemblance en termes de caractéristiques des élevages et de leurs conduites.

\section{Références}

Alary V, Moulin CH, Lasseur J, Aboul-Naga A, Sraïri MT. 2019. The dynamic of crop-livestock systems in the Mediterranean and future prospective at local level: A comparative analysis for South and North Mediterranean systems. Livestock Science 224: 40-49. DOI: 10.1016/j.livsci.2019.03.017

Amoudry JP. 2002. Rapport d'information fait au nom de la mission commune d'information chargée de dresser un bilan de la politique de la montagne et en particulier de l'application de la loi du 9 janvier 1985, de son avenir, et de ses nécessaires adaptations. Paris, France: Senat, ${ }^{\circ} 15$, tome 1, 408 p. http://www.senat.fr/ notice-rapport/2002/r02-015-1-notice.html.

Benjelloun B. 2013. L'élevage ovin dans les montagnes marocaines comme vecteur de développement économique : cas de la province d'Azilal. Options Méditerranéennes 108: 267-272.

Boughalmi A, Araba A, Yessef M. 2015. Dynamics of extensive sheep production systems in Morocco. Livestock Research for Rural Development 27(11).

Bourbouze A. 1999. Gestion de la mobilité et résistance des organisations pastorales des éleveurs du Haut Atlas marocain face aux transformations du contexte pastoral maghrébin. In: NiamirFuller M, FAO and Beijer, eds. Managing mobility in African rangelands: The legitimization of transhumance. International Institute of Ecological Economics, IT Publication, pp. 236-265. DOI: $10.3362 / 9781780442761.010$. 
Bourbouze A. 2006. Systèmes d'élevage et production animale dans les steppes du nord de l'Afrique: une relecture de la société pastorale du Maghreb. Secheresse 17(1-2): 31-39.

Casasús I, Jozo R, Andrea R. 2012. Animal farming and environmental interactions in the Mediterranean Region. EAAP Publication 131: 30. DOI: 10.3920/978-90-8686-741-7.

Centre régional d'investissement de Béni Mellal-Khénifra. 2015. Monographie de la région Béni Mellal-Khénifra. Maroc : Ministère de l'Intérieur, Direction Générale des Collectivités Locales, $56 \mathrm{p}$.

CIRAD-GRET. 2009. Mémento de l'Agronome. Paris (France) : Editions Quae, 447 p.

Direction provinciale de l'agriculture d'Azilal. 2019. Monographie de la zone d'action de la DPA. Maroc.

El Amiri B, Chentouf M, Chriyaa A. 2007. L'élevage caprin dans la vallée d'Ait Bouguemaz: diagnostic, voies d'améliorations et perspectives de recherche. Technical Report, Bulletin de Transfert de Technologie en Agriculture $\mathrm{n}^{\mathrm{o}}$ 154. Maroc: Ministère de l'Agriculture et de la Pêche Maritime, 4p. DOI: 10.13140/ 2.1.1898.5763.

FAO. 2010. Système d'exploitation agricole mixte de haute altitude (Andes centrales). Rome (Italie) : FAO. Disponible sur http://www. fao.org/3/Y1860f/y1860f19.htm.

Gavinet J. 2007. Appropriation, usage et gestion des ressources sylvopastorales à Wabzaza, Haut Atlas Central, Maroc. Mémoire de fin d'études. Paris (France): Agroparitech, $106 \mathrm{p}$.

Gibon A, Balent G, Alard D, Muntané y Raich J, Ladet S, et al. 2004. L'usage de l'espace par les exploitations d'élevage de montagne et la gestion de la biodiversité. Fourrages 178: 245-263.

Ibnelbachyr M, Mounsif M. 2011. Fragilité des modes de conduite des troupeaux de petits ruminants en zones de montagne. Cas du bassin versant de l'Oued Lakhdar (Maroc). Options Méditerranéennes 100: $157-161$.

Jemaa T, Huguenin J, Moulin C-H, Najar T. 2016. Les systèmes d'élevage de petits ruminants en Tunisie centrale: stratégies différenciées et adaptations aux transformations du territoire. Cahiers Agricultures 25: 45005. DOI: 10.1051/cagri/2016030.

Kauffman JC. 1996. Semi-directive interviews. Collection Sociologie 128. Paris (France) : Éditions Nathan Université.
Martin B, Lherm M, Béranger C. 2014. Evolutions et perspectives de l'élevage des ruminants dans les montagnes françaises. INRA Productions Animales 27(1): 5-16. DOI: 10.20870/productionsanimales.2014.27.1.3050.

Ministère de l'Agriculture et de la Pêche Maritime. 2014. Deuxième rapport national sur l'état des ressources génétiques animales. http://www.fao.org/3/i4787e/i4787f27.pdf [consulté le 14/01/ 2020].

Ministère de l'Agriculture et de la Pêche Maritime. 2014. Strategie de developpement des petits ruminants dans le cadre du plan Maroc vert. IAV Hassan II. Disponible sur https://docplayer.fr/40027813Strategie-de-developpement-des-petits-ruminants-dans-le-cadredu-plan-maroc-vert.html [consulté le 17/01/2020].

Ministère de l'Agriculture, de la Pêche Maritime, du Développement Rural et des Eaux et Forêts. 2017. Statistiques de l'élevage, campagne agricole 2015-2016.

Nassif F, El Amiri B, Cohen A. 2011. L'élevage caprin à viande au Maroc, opportunités et perspectives (Cas du caprin d'Ait Bazza). Transfert de technologies en Agriculture 61: 1-7. https://www. researchgate.net/publication/234081795.

Nettier B, Dobremez L, Coussy J L, Romagny T. 2010. Attitudes of livestock farmers and sensitivity of livestock farming systems to drought conditions in the French Alps. Journal of Alpine Research 98(4). DOI: 10.4000/rga.1307.

Nettier B, Dobremez L, Brunschwig G. 2015. «Prendre en compte les interactions entre alpages et exploitations dans les systèmes agropastoraux: une revue bibliographique». INRA Productions Animales 28(4): 329-340. DOI: 10.20870/productions-anima les.2015.28.4.3037.

R-Development-core-team. 2019. A Language and Environment for Statistical Computing, Version 3.6.2.

Sraïri MT, Benabdeljelil K, Touré A. 2003. Typologie d'exploitations agricoles en zone montagneuse au Maroc à travers l'analyse des activités d'élevage. New. Medit 4.

Srour G. 2006. Amélioration durable de l'élevage des petits ruminants au Liban. Thèse de doctorat. France: Institut National Polytechnique de Lorraine, $291 \mathrm{p}$.

Citation de l'article : El Aayadi S, Araba A, Jouven M. 2020. Impact de l'altitude sur les caractéristiques des systèmes d'élevage extensifs dans le Haut Atlas central, Maroc. Cah. Agric. 29: 33. 